\title{
Correlación entre materia grasa, azúcares reductores y humedad en la pulpa de aceitunas.
}

\author{
Por A.H. Sánchez Gómez y M. J. Fernández Díez \\ Instituto de la Grasa y sus Derivados. Apartado 1078 - 41012 SEVILLA
}

\section{RESUMEN}

Correlación entre materia grasa, azúcares reductores y humedad en la pulpa de aceitunas.

En aceitunas de dos variedades españolas, Gordal y Zorzaleña, y una de origen griego, Conservolea, se realiza un análisis estadístico de los resultados obtenidos durante el desarrollo y maduración del fruto, en la determinación de los componentes mayoritarios de la pulpa fresca, materia grasa, azúcares reductores y humedad.

Se obtiene una ecuación global de regresión múltiple, no encontrándose diferencias significativas atribuibles a la variedad, campaña, y región consideradas en el estudio.

PALABRAS-CLAVE: Aceituna (pulpa) - Azúcares reductores - Humedad - Materia grasa - Regresión múltiple.

\section{SUMMARY}

Correlation among fat, reducing sugars and moisture contents in the fresh pulp of olives.

Statistical analysis of the results obtained for fat, reducing sugars, and moisture, in the fresh pulp of olives from two Spanish varieties, Gordal and Zorzaleña, and one of Greek origin, Conservolea, during the growth and ripening period, gives rise to a multiple regression equation, which is valid for the three considered varieties.

No significant differences, related to variety, crop, and culture region, were found in this study.

KEY-WORDS: Fat - Moisture - Multiple regression - Olive (pulp) - Reducing sugars.

\section{INTRODUCCION.}

El fruto del olivo cultivado (Olea europaea sativa, Hoffg, Link) desempeña en la industria alimentaria dos misiones fundamentales: como materia prima para la obtención de su aceite, sumamente apreciado en el comercio internacional, por sus características organolépticas especiales y su valor nutritivo y biológico (1), y como fruto aderezado y fermentado que constituye un alimento bastante completo, y fundamentalmente uno de los aperitivos mas universalmente conocido y difundido (2) (3).

Entre sus componentes mayoritarios hemos de destacar la materia grasa, cuya extracción es el objetivo principal de una gran parte del cultivo, y los azúcares en general, como constituyentes esenciales para la fermentación a que se somete el resto de la producción, durante su elaboración como aceituna de mesa. Dentro de los mismos, la principal aportación es la que corresponde a los azúcares reductores simples (4), y a éstos nos referiremos exclusivamente en el resto del trabajo.

Las distintas variedades de estos frutos pueden, en principio, destinarse a cumplir ambos objetivos, y es precisamente su composición la determinante del valor económico comercial en cada caso. Es lógico que para el primer uso (extracción) deberán seleccionarse principalmente aquellas variedades que presenten un alto contenido en aceite, y que, para el consumo directo (aceituna de mesa) se elijan las que manifiesten, junto con otras características fisico-químicas, un sustancial contenido de materia fermentable. Por supuesto, la mayor o menor cantidad de ambos componentes presente en la pulpa depende, además de la variedad, de múltiples factores, entre los que se han de destacar el estado de desarrollo y madurez en el período de recolección, las condiciones climáticas, y el sistema de cultivo (secano o regadío), pudiendo todos ellos influir muy sensiblemente en el grado de humedad del fruto en cuestión.

Los constituyentes mayoritarios mencionados, materia grasa, azúcares reductores y humedad, que representan en conjunto alrededor del $90 \%$ de la pulpa comestible, tienen necesariamente que estar correlacionados entre sí, de acuerdo con los principios generales de la lipogénesis, considerando la buena correlación simple que presenta la primera con respecto a cada uno de los otros dos.

El objeto de este trabajo es, simplemente, comprobar si existe algún tipo de relación matemática sencilla entre los tres componentes citados para distintas variedades durante el proceso de desarrollo y maduración del fruto. En caso afirmativo se analizará si la correlación múltiple se cumple para el conjunto de las variedades estudiadas y 
cuál es su grado de exactitud para predecir el contenido de uno de los componentes de interés comercial (materia grasa o azúcares reductores) a partir de los valores conocidos para los otros.

\section{PARTE EXPERIMENTAL.}

\subsection{Muestras utilizadas.}

Para este estudio se emplean dos series de datos de investigaciones experimentales previas, correspondientes a frutos de procedencia española (5) y griega (6) respectivamente. La primera, está constituida por veintitrés muestras de aceitunas de dos variedades, de características muy diferentes entre sí: Gordal o Sevillana (15 muestras), de bajo contenido en aceite, piel y pulpa delicada, frutos de gran tamaño y tradicionalmente utilizada como aceituna de mesa; y Zorzaleña (8 muestras), de alto contenido en aceite, textura más resistente, menor tamaño medio, y emplea- das casi exclusivamente para la extracción del aceite. Las muestras duplicadas, se recolectan de árboles marcados, durante el período de desarrollo y maduración del fruto, y corresponden a distintas cosechas y a la misma finca.

Para la determinanción de azúcares reductores se emplea el método clásico volumétrico de Lane-Eynon; el contenido de materia grasa se obtiene por extracción en Soxhlet, con éter etílico, de la pulpa previamente desecada; y la humedad por desecación, hasta peso constante, en estufa.

Los datos de la serie griega corresponden a diecinueve muestras de la variedad denominada Conservolea, de características fisico-químicas que pueden considerarse, en general, como intermedias entre las dos españolas seleccionadas, y procedentes de dos regiones que presentan condiciones ambientales distintas, Agios Constantinos (11 muestras) y Agria (8 muestras). Provienen igualmente de árboles marcados, y cubren en ambos casos el ciclo de desarrollo y maduración del fruto en la misma cosecha. Las determinaciones de

Tabla 1

Composición química. Variedades Gordal y Zorzaleña.

\begin{tabular}{|c|c|c|c|c|c|}
\hline \multirow[b]{2}{*}{ Año } & \multirow[b]{2}{*}{$\begin{array}{l}\text { Fecha de } \\
\text { recolección }\end{array}$} & \multicolumn{4}{|c|}{ \& Pulpa fresca } \\
\hline & & Materia grasa & Azúcares & s reductores & Humedad \\
\hline \multicolumn{6}{|c|}{ GORDAL } \\
\hline 1950 & $\begin{array}{rl}3 & \text { Julio } \\
28 & " \\
5 & \text { Septiem. } \\
6 & \text { Octubre }\end{array}$ & $\begin{array}{r}0,60 \\
1,15 \\
10,70 \\
12,75\end{array}$ & & $\begin{array}{l}7,28 \\
7,84 \\
6,33 \\
4,88\end{array}$ & $\begin{array}{l}69,00 \\
74,20 \\
68,85 \\
70,33\end{array}$ \\
\hline 1951 & $\begin{array}{rl}26 & \text { Junio } \\
16 & \text { Julio } \\
6 & \text { Septiem. } \\
22 & \text { Octubre } \\
14 & \text { Noviemb. } \\
17 & " \\
28 & "\end{array}$ & $\begin{array}{r}0,70 \\
1,60 \\
6,25 \\
16,10 \\
18,60 \\
18,95 \\
19,50\end{array}$ & & $\begin{array}{l}7,58 \\
7,81 \\
5,96 \\
4,30 \\
3,66 \\
3,40 \\
3,08\end{array}$ & $\begin{array}{l}72,65 \\
76,20 \\
73,73 \\
68,05 \\
68,10 \\
67,25 \\
69,60\end{array}$ \\
\hline 1952 & $\begin{array}{ll}5 & \text { Agosto } \\
4 & \text { Septiem. } \\
4 & \text { Octubre } \\
6 & \text { Noviemb. }\end{array}$ & $\begin{array}{r}4,61 \\
8,75 \\
16,01 \\
18,53\end{array}$ & & $\begin{array}{l}5,61 \\
4,47 \\
4,31 \\
3,50\end{array}$ & $\begin{array}{l}76,70 \\
71,73 \\
68,00 \\
68,60\end{array}$ \\
\hline \multicolumn{6}{|c|}{ ZORZALEÑA } \\
\hline 1951 & $\begin{aligned} 26 & \text { Junio } \\
16 & \text { Julio } \\
6 & \text { Septiem. } \\
28 & \text { Noviemb. }\end{aligned}$ & $\begin{array}{r}0,65 \\
6,38 \\
17,70 \\
26,50\end{array}$ & & $\begin{array}{l}8,75 \\
8,20 \\
5,87 \\
3,40\end{array}$ & $\begin{array}{l}68,93 \\
68,35 \\
60,55 \\
59,45\end{array}$ \\
\hline 1952 & $\begin{array}{l}5 \text { Agosto } \\
4 \text { Septiem. } \\
4 \text { Octubre } \\
6 \text { Noviemb. }\end{array}$ & $\begin{array}{l}11,35 \\
17,55 \\
23,40 \\
29,01\end{array}$ & & $\begin{array}{l}5,97 \\
5,03 \\
4,31 \\
3,47\end{array}$ & $\begin{array}{l}65,70 \\
62,72 \\
54,99 \\
53,00\end{array}$ \\
\hline
\end{tabular}


azúcares reductores, materia grasa, y humedad en la pulpa fresca, se realizan por los autores por los mismos métodos citados anteriormente.

\subsection{Análisis estadístico.}

Se utiliza el paquete de programas estadístico SPSS/PC+ (7) para los análisis de regresión y varianza realizados.

\section{RESULTADOS Y DISCUSIÓN.}

En la Tabla I se dan los resultados analíticos obtenidos para las dos variedades españolas estudiadas, expresados como porcentaje de la pulpa húmeda, valores que se utilizan en todos los cálculos posteriores. La Tabla II muestra los valores correspondientes a la variedad griega, en las dos regiones consideradas.

Tabla II

Composición química. Variedad Conservolea.

\begin{tabular}{|c|c|c|c|c|c|c|c|}
\hline \multirow[b]{2}{*}{ Año } & \multirow{2}{*}{\multicolumn{2}{|c|}{$\begin{array}{l}\text { Fecha de } \\
\text { recolección }\end{array}$}} & \multicolumn{5}{|c|}{ \& Pulpa fresca } \\
\hline & & & \multicolumn{2}{|c|}{ Materia grasa } & \multicolumn{2}{|c|}{ Azúcares reductores } & \multirow[t]{2}{*}{ Humedad } \\
\hline \multicolumn{7}{|c|}{ AGIOS CONSTANTINOS } & \\
\hline 1969 & $\begin{array}{r}13 \\
24 \\
3 \\
14 \\
29 \\
8 \\
30 \\
17 \\
7 \\
30 \\
29\end{array}$ & $\begin{array}{l}\text { Julio } \\
\text { Agosto } \\
\text { " } \\
\text { Septiem. } \\
\text { Octubre } \\
\text { Noviemb. } \\
\text { " Diciemb. }\end{array}$ & $\begin{array}{r}1,12 \\
3,06 \\
4,12 \\
7,60 \\
9,10 \\
10,38 \\
15,58 \\
19,25 \\
21,52 \\
24,15 \\
24,25\end{array}$ & & & $\begin{array}{l}6,34 \\
5,71 \\
5,63 \\
4,90 \\
4,97 \\
4,60 \\
4,03 \\
3,41 \\
3,04 \\
3,62 \\
2,23\end{array}$ & $\begin{array}{l}76,73 \\
74,00 \\
72,75 \\
71,50 \\
70,50 \\
70,75 \\
70,25 \\
69,00 \\
65,00 \\
61,40 \\
59,50\end{array}$ \\
\hline \multicolumn{8}{|c|}{ AGRIA } \\
\hline 1969 & $\begin{array}{l}11 \\
31 \\
11 \\
24 \\
18 \\
27 \\
27 \\
28\end{array}$ & $\begin{array}{l}\text { Julio } \\
" \\
\text { Agosto } \\
" \\
\text { Septiem. } \\
\text { Octubre } \\
\text { Noviemb. } \\
\text { Diciemb. }\end{array}$ & $\begin{array}{r}1,22 \\
3,62 \\
6,84 \\
8,57 \\
12,50 \\
18,15 \\
23,48 \\
23,75\end{array}$ & & & $\begin{array}{l}6,37 \\
5,87 \\
5,04 \\
3,93 \\
3,94 \\
3,13 \\
2,71 \\
2,35\end{array}$ & $\begin{array}{l}76,00 \\
74,30 \\
71,80 \\
72,20 \\
71,50 \\
66,00 \\
61,20 \\
58,50\end{array}$ \\
\hline
\end{tabular}

3.1. Correlación entre materia grasa - azúcares y materia grasa - humedad.

En la Tabla III se recogen para cada una de las variedades estudiadas las ecuaciones obtenidas para la estimación de la materia grasa en función del contenido en azúcares o del contenido en humedad.

Se observa que los coeficientes de correlación son mayores para el caso de la regresión frente a los azúcares que frente a la humedad, aunque cuando se considera globalmente el conjunto de todos los datos ocurre lo contrario.

3.2. Correlación múltiple materia grasa azúcares y humedad.

En la Tabla IV se recogen, para cada una de las variedades estudiadas, las ecuaciones obteni- das para la materia grasa en función de los azúcares y la humedad. Como se observa de la comparación con las regresiones simples, los coeficientes de correlación son ligeramente superiores y, para el caso de considerar de modo conjunto todos los datos, son claramente mayores.

También se realiza un análisis de varianza para determinar si es posible utilizar una ecuación conjunta para las tres variedades, en lugar de una individual para cada una de ellas. En la Tabla $V$ se recoge dicho análisis y el estadístico $F$ obtenido es de 2,11, menor que el tabulado para un nivel de significación de $0,05, F(6,33)=2,50$, por lo que se puede concluir que no existen diferencias significativas entre las ecuaciones para cada una de las tres variedades, proponiéndose, por tanto, para estimar la cantidad de materia grasa 
Tabla III

Correlaciones simples de la materia grasa frente al contenido en azúcares reductores o de humedad.

A) AZUCARES REDUCTORES

\begin{tabular}{|c|c|c|c|c|c|c|}
\hline \multirow[b]{2}{*}{ Variedad } & \multirow[b]{2}{*}{$\begin{array}{r}\text { Número } \\
\text { datos }\end{array}$} & \multicolumn{2}{|c|}{ Azúcar } & Térm. & indepen. & \multirow[b]{2}{*}{$\begin{array}{l}\text { Coeficiente } \\
\text { correlación }\end{array}$} \\
\hline & & Coef. & $\begin{array}{l}\text { Desviac. } \\
\text { estandar }\end{array}$ & Coef. & $\begin{array}{l}\text { Desviac. } \\
\text { estandar }\end{array}$ & \\
\hline GORDAL & 15 & $-4,09$ & 0,40 & 32,13 & 2,22 & $-0,9438$ \\
\hline ZORZAL. & 8 & $-4,81$ & 0,44 & 43,64 & 2,59 & $-0,9762$ \\
\hline CONSERV. & 19 & $-6,07$ & 0,47 & 38,70 & 2,11 & $-0,9527$ \\
\hline GLOBAL & 42 & $-4,24$ & 0,44 & 33,41 & 2,27 & $-0,8374$ \\
\hline
\end{tabular}

B) HUMEDAD

\begin{tabular}{|c|c|c|c|c|c|c|}
\hline \multirow[b]{2}{*}{ Variedad } & \multirow[b]{2}{*}{$\begin{array}{l}\text { Número } \\
\text { datos }\end{array}$} & \multicolumn{2}{|c|}{ Humedad } & Térm. & indepen. & \multirow[b]{2}{*}{$\begin{array}{l}\text { Coeficiente } \\
\text { correlación }\end{array}$} \\
\hline & & Coef. & $\begin{array}{l}\text { Desviac. } \\
\text { estandar }\end{array}$ & Coef. & $\begin{array}{l}\text { Desviac. } \\
\text { estandar }\end{array}$ & \\
\hline GORDAL & 15 & $-1,76$ & 0,44 & 135,12 & 31,11 & $-0,7441$ \\
\hline ZORZAL. & 8 & $-1,58$ & 0,24 & 114,13 & 15,06 & $-0,9358$ \\
\hline CONSERV. & 19 & $-1,43$ & 0,12 & 111,15 & 8,33 & $-0,9447$ \\
\hline GLOBAL & 42 & $-1,23$ & 0,12 & 96,71 & 8,31 & $-0,8491$ \\
\hline
\end{tabular}

Tabla IV

Correlación múltiple de la materia grasa frente al contenido en azúcares reductores y humedad.

\begin{tabular}{|c|c|c|c|c|c|c|c|c|}
\hline \multirow[b]{2}{*}{ Variedad } & \multirow[b]{2}{*}{$\begin{array}{l}\text { Número } \\
\text { datos }\end{array}$} & \multicolumn{2}{|c|}{ AZUCARES REDUCTORES } & \multicolumn{2}{|c|}{ HUMEDAD } & \multicolumn{2}{|c|}{ TERMINO INDEPEND. } & \multirow[b]{2}{*}{$\begin{array}{l}\text { Coeficiente } \\
\text { correlación }\end{array}$} \\
\hline & & Coeficiente & $\begin{array}{l}\text { Desviac. } \\
\text { estandar }\end{array}$ & Coeficiente & $\begin{array}{l}\text { Desviac. } \\
\text { estandar }\end{array}$ & Coeficiente & $\begin{array}{l}\text { Desviac. } \\
\text { estandar }\end{array}$ & \\
\hline GORDAL & 15 & $-3,43$ & 0,45 & $-0,56$ & 0,25 & 68,20 & 16,03 & 0,9610 \\
\hline ZORZAL. & 8 & $-3,35$ & 0,70 & $-0,57$ & 0,24 & 70,42 & 11,47 & 0,9888 \\
\hline CONSERV. & 19 & $-3,44$ & 0,88 & $-0,69$ & 0,21 & 74,94 & 11,11 & 0,9721 \\
\hline GLOBAL & 42 & $-2,74$ & 0,25 & $-0,82$ & 0,07 & 82,01 & 4,36 & 0,9654 \\
\hline
\end{tabular}

en función del contenido en azúcares reductores y de la humedad, la ecuación global:

$$
G=-0,82 \mathrm{H}-2,74 \mathrm{~A}+82,01
$$

en la que $G$ representa la materia grasa estimada, y $\mathrm{H}$ y $\mathrm{A}$ los contenidos en humedad y azúcares reductores, respectivamente, expresados todos los datos como porcentaje de la pulpa fresca.
Otro estudio, similar al anterior, pero realizado dentro de cada variedad, para determinar la posible influencia de las distintas campañas en las variedades Gordal y Zorzaleña, y de las dos regiones estudiadas en la variedad Conservolea, permite concluir que, en ninguno de los casos estudiados, existe una influencia significativa de la campaña, ni de la regiónconsiderada. No obstante, para poder 
Tabla V

Análisis de varianza para la comparación entre variedades

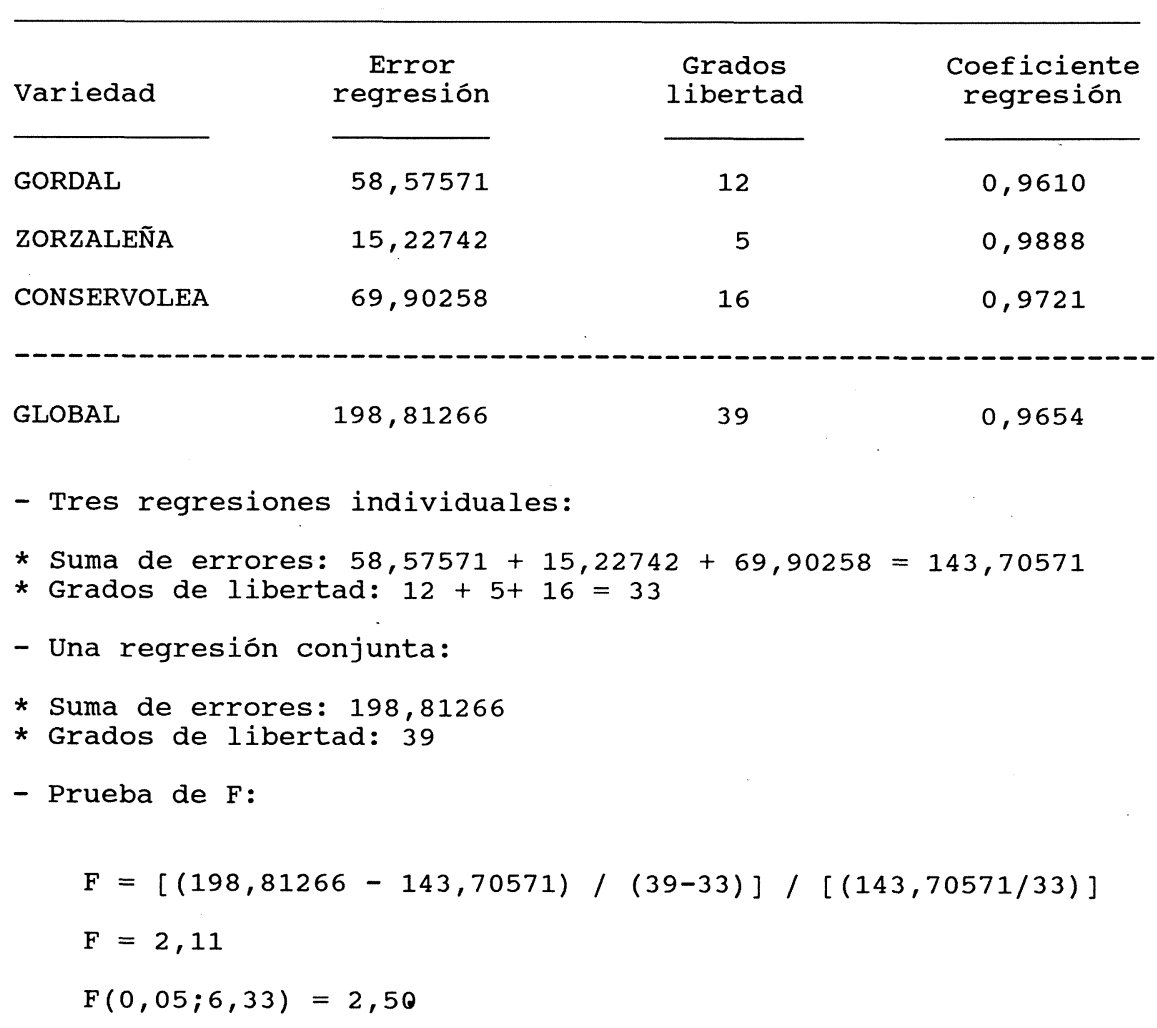

Tabla VI

Valores estimados de materia grasa, para todas las variedades, regiones de cultivo y campañas analizadas.

\begin{tabular}{|c|c|c|c|}
\hline \multirow[b]{2}{*}{ Real (Y) } & \multicolumn{2}{|c|}{ Materia grasa (\% pulpa fresca) } & \multirow[b]{2}{*}{ Estimada (X) } \\
\hline & Estimada $(\mathrm{X})$ & Real (Y) & \\
\hline 0,60 & 5,50 & 12,50 & 12,56 \\
\hline 0,65 & 1,54 & 12,75 & 10,96 \\
\hline 0,70 & 1,68 & 15,58 & 13,34 \\
\hline 1,12 & 1,72 & 16,01 & 14,42 \\
\hline 1,15 & $-0,30$ & 16,10 & 14,41 \\
\hline 1,22 & 2,24 & 17,55 & 16,79 \\
\hline 1,60 & $-1,86$ & 17,70 & 16,27 \\
\hline 3,06 & 5,68 & 18,15 & 19,29 \\
\hline 3,62 & 5,00 & 18,53 & 16,14 \\
\hline 4,12 & 6,93 & 18,60 & 16,12 \\
\hline 4,61 & 3,74 & 18,95 & 17,52 \\
\hline 6,25 & 5,22 & 19,25 & 16,06 \\
\hline 6,38 & 3,52 & 19,50 & 16,47 \\
\hline 6,84 & 9,31 & 21,52 & 20,35 \\
\hline 7,60 & 9,94 & 23,40 & 25,09 \\
\hline 8,57 & 12,01 & 23,48 & 24,37 \\
\hline 8,75 & 11,42 & 23,75 & 27,56 \\
\hline 9,10 & 10,57 & 24,15 & 21,72 \\
\hline 10,38 & 11,38 & 24,25 & 27,07 \\
\hline 10,70 & 8,21 & 26,50 & 23,92 \\
\hline 11,35 & 11,78 & 29,01 & 29,02 \\
\hline
\end{tabular}


estudiar de modo exhaustivo estos efectos, sería necesario disponer de un número de datos más elevado.

\subsection{Correlación entre valores reales y} estimados.

Los valores reales de materia grasa y los estimados a partir de la ecuación general de regresión múltiple, guardan entre sí una correlación simple muy significativa $(r=0,9654)$, respondiendo a la ecuación de regresión $Y=X+0,01$, frente a la teórica $Y=X$, en la que $Y$ son los valores reales de materia grasa y $X$ los estimados a partir de las cifras de azúcares reductores y humedad (Tabla VI).

\section{CONCLUSION.}

El contenido en materia grasa de la pulpa de aceitunas muestra una correlación múltiple con los porcentajes de azúcares reductores y humedad presentes en la misma, con un alto grado de significación, al menos para las tres variedades estudiadas, durante el ciclo completo de desarrollo y maduración del fruto.

La ecuación de regresión múltiple que liga los constituyentes mayores citados permite, únicamente, estimar datos orientativos.

\section{BIBLIOGRAFIA.}

1. Mataix Verdú, F. J. y Martínez de Victoria Muñoz, E.- "El aceite de oliva. Bases para el futuro".- p. 76-117.- Junta de Andalucia. Consejeria de Agricultura y Pesca.- Sevilla, 1988.

2. Fernández Díez, M. J.- "Importance of Research on Nutritional Value of Table Olives".- Proceedings of the III International Congress on the Biological Value of Olive Oil.p. 471-484.- Canea, Crete (Greece), 1980.

3. Balatsouras, G.- "Nutritive and Biological Value of Greek Table Olives".- Proceedings of the III International Congress on the Biological Value of Olive Oil.- p. 485-520.- Canea, Crete (Greece), 1980.

4. Fernández Díez, M. J.; Castro Ramos, R. de; Garrido Fernández, A.; González Cancho, F.; González Pellissó, F.; Nosti Vega, M.; Heredia Moreno, A.; Mínguez Mosquera, M. I.; Rejano Navarro, L.; Durán Quintana, M. C.; Sánchez Roldán, F.; García García, P.; y Castro Gómez-Millán, A.- "Biotecnología de la aceituna de mesa".- p.27-38:-C. S. I. C. Instituto de la Grasa y sus Derivados. Madrid. Sevilla, 1985.

5. Rodriguez de la Borbolla y Alcalá, J. M.; Fernández Díez, M. J.; y González Pellisó, F.- "Cambios en la composición de la aceituna durante su desarrollo.I.-" Grasas y Aceites 6 (1955) 5-22.

6. Alygizakis, E. M. y Patroclos, G. A.- "Contribution a l'etude des changements survenant dans la composition chimique de l'olive de la varieté Conservolia au cours de sa croissanse et de sa maturation".- Compte rendu de la III Conférence Internationale des Techniciens Oléicoles, Conm.Chi 8.p 57. Torremolinos, Espagne, 1971.

7. Norusis, M. J.- "SPSS-X Advanced Statistics Guide".- Mc Graw-Hill Book Company. New York, 1985.

(Recibido: Febrero 1991) 